\title{
PRINCIPIA IURIS: CARACTERIZACIÓN DE UNA TEORÍA JURÍDICA *
}

\author{
Marina Gascón Abellán \\ Universidad de Castilla-La Mancha (UCLM)
}

RESUMEN. La teoría del Derecho que L. FerRAJolı nos presenta en Principia iuris puede ser caracterizada por tres rasgos: por el objeto sobre el que versa (o sea, por su dimensión semántica); por su finalidad u objetivo (o sea, por su dimensión pragmática); y por el método que utiliza para su construcción (esto es, por su dimensión sintáctica). Atendiendo a su dimensión semántica, se trata de una teoría del Derecho amplia y elástica (porque permite reconstruir, a medida de las necesidades, casi cualquier sistema jurídico) y normativista y realista al mismo tiempo (porque abarca las dimensiones normativa y fáctica del fenómeno jurídico). Atendiendo a su dimensión pragmática, la finalidad u objetivo central de la teoría es redefinir el paradigma teórico y normativo del Estado constitucional de Derecho a fin de que resulten muy claros sus principios y bien visibles las divergencias entre las prácticas jurídicas y dichos principios. Pero la novedad principal de Principia iuris es la utilización del método axiomático en su reconstrucción, lo que permite una mayor transparencia y control lógico de las tesis que contiene.

Palabras clave: Ferrajoli, Principia iuris, teorías del Derecho normativistas y realistas, Estado constitucional de Derecho, método axiomático.

ABSTRACT. The Principia luris theory of law could be distinguished by the following characteristic features: by the subject matter it deals with (i. e. semantic aspect); by its purpose (i.e. pragmatic aspect); and by the method used to construct it (i. e. syntactic aspect). Regarding the semantic point of view it can be described as being both a wide and elastic theory of law (due to the fact that it can be used to reconstruct almost any legal system) as well as a normativist and realist one (given that it embraces the normative and the factual aspects of the legal phenomenon). In the pragmatic aspect, the main purpose of the theory is to redefine the theoretical and normative paradigm of the constitutional rule of law, in order to make both its principles and the divergences between the legal practices and these principles very clear. But the prime novelty of Principia luris is that it uses the axiomatic method in its reconstruction, thus allowing a clearer and more logical control of its theses.

Keywords: FerRajol, Principia luris, normativist and realist theories of law, constitutional rule of law, axiomatic method.

* Fecha de recepción: 1 de diciembre de 2008. Fecha de aceptación: 18 de diciembre de 2008. 


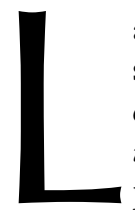

a teoría del Derecho que L. FerRajoli nos presenta en Principia Iuris puede ser caracterizada — como hace el autor en el Prefacio_- por tres rasgos: por el objeto sobre el que versa (o sea, por su dimensión semántica); por su finalidad u objetivo (o sea, por su dimensión pragmática); y por el método que utiliza - para su construcción (esto es, por su dimensión sintáctica). Muy resumidamente, puede caracterizarse por el modo en que responde a estas tres preguntas: ¿de qué habla la teoría?; ¿a qué fin sirve la teoría o cómo se justifica?; y ¿cómo se construye? Las respuestas a estas tres preguntas constituyen, pues, tres posiciones metateóricas sobre la semántica, la pragmática y la sintáctica de la teoría del derecho.

\section{LA SEMÁNTICA DE LA TEORÍA}

La metateoría semántica del Derecho de Principia Iuris (es decir, la que establece la semántica de la teoría: de qué habla la teoría o cuál es su objeto o campo de análisis) permite caracterizar a la teoría del Derecho como amplia y elástica -atendiendo a su extensión- y como normativista y realista al mismo tiempo - atendiendo a su intensión. En lo referente a su extensión ello es así porque, aunque el objeto preferente de la teoría es el derecho positivo moderno, puede decirse que la teoría habla casi de cualquier sistema jurídico, por simple o complejo que éste sea; y en lo referente a su intensión ello es así porque abarca las dos dimensiones del fenómeno jurídico, la normativa y la fáctica, las normas y los hechos.

\subsection{La extensión de la teoría: su elasticidad}

El anhelo de toda teoría jurídica mínimamente ambiciosa —y la de FerRAJOLI sin duda lo es- es poder dar cuenta no de uno sino de muchos (o de todos los) sistemas jurídicos, y ello, naturalmente, sin restarle capacidad explicativa de los fenómenos más característicos y complejos de los sistemas jurídicos más evolucionados. Es evidente, sin embargo, que la extensión de una teoría (es decir, su capacidad de aplicación a muchos objetos) varía inversamente a su intensión (es decir, a su capacidad de dar cuenta de los aspectos más complejos de los ordenamientos más evolucionados). Por eso la aspiración teórica a la máxima extensión plantea un problema: una teoría cuyos conceptos estén dotados de una gran extensión será aplicable a un mayor número de sistemas jurídicos pero su capacidad explicativa de los sistemas jurídicos más complejos será menor; y a la inversa, una teoría cuyos conceptos sean complejos y/o estén dotados de una gran intensión tendrá una gran capacidad explicativa de los sistemas jurídicos muy evolucionados, pero será aplicable a menos sistemas jurídicos. Para superar este escollo FERRAJOLI proporciona una solución talentosa: opta por una teoría que comienza introduciendo conceptos elementales, dotados de poca intensión (como permiso, obligación, prohibición, norma jurídica, etc.) y que permitirían reconstruir los sistemas jurídicos primitivos e incluso sistemas normativos no jurídicos; y continúa introduciendo conceptos complejos, dotados cada vez de mayor intensión (como derechos fundamentales, acto constituyente, constitución, separación de poderes, garantías, etc.), que permitirían reconstruir, sucesivamente, los sistemas jurídicos positivos y los sistemas jurídicos 
del Estado legal de Derecho y del Estado constitucional de Derecho ${ }^{1}$. Por eso no tiene demasiado sentido hablar del alcance empírico global de esta teoría del Derecho, sino que habría que hablar más bien del alcance más o menos amplio de sus particulares conceptos, tesis y subteorías². Y por eso la teoría del Derecho de Principia Iuris puede calificarse, a mi juicio, no sólo como amplia sino también y sobre todo como elástica, pues permite reconstruir e interpretar, a medida de las necesidades, sistemas jurídicos en distinto grado de evolución y complejidad.

\subsection{La intensión de la teoría: normativista y realista}

Desde el punto de vista de la intensión de la teoría se plantea la cuestión (metateórica) de si su objeto es la dimensión normativa del Derecho o su dimensión fáctica; y en particular - y conectado con lo anterior- se plantea el asunto de si la teoría del Derecho tiene o no una existencia autónoma frente a la dogmática jurídica y a la sociología del Derecho e incluso frente a la filosofía política o filosofía de la justicia. FERRAJOLI resuelve esta cuestión mediante una posición metateórica que impone a la teoría la «integración» de las dos dimensiones del Derecho mencionadas y que, derivadamente, marca su distinción frente a los discursos de la dogmática y la sociología jurídica: es necesario que la teoría, en la medida en que ha de ser apta para reconstruir también (y sobre todo) el Derecho del Estado constitucional, dé cuenta de la eventual e inevitable divergencia entre el ser y el deber ser en el Derecho que constituye la columna de ese paradigma, y por consiguiente es necesario que dé cuenta tanto de la dimensión normativa del Derecho como de su dimensión fáctica. Si puede decirse así, la divergencia entre el «ser» y el «deber ser», que la teoría del garantismo (o del estado constitucional) había llevado hasta la esfera misma del Derecho ${ }^{3}$, es llevada ahora también a la teoría del derecho, superándose de este modo la aproximación al Derecho puramente normativista y la puramente realista.

En efecto, en los sistemas complejos que la teoría debe poder explicar, como son los Estados constitucionales de Derecho, no basta con una aproximación al Derecho puramente normativista o puramente realista, porque ambas aproximaciones ofrecen una representación del Derecho que resulta parcial y por tanto falaz. La aproximación normativista es parcial o incompleta porque da cuenta del Derecho que (normativamente) «debe ser» pero ignora el Derecho que es; o sea, su práctica efectiva. Ignora por

1 2007: Principia Iuris. Teoria del diritto e della democracia, Roma-Bari: Laterza, vol. I, Teoria del diritto, esp. pp. 6 y 20. La teoría del Derecho elaborada aquí — precisa FERRAJOLI— «quiere ser una teoría del Derecho positivo moderno y de las formas jurídicas de la democracia. Su parte tercera está dedicada al "Estado de Derecho" (...) Su parte cuarta se dirige a explicar las complejas formas y garantías de la "democracia constitucional" (...) La primera parte de la teoría, dedicada a la deóntica, y una parte de la segunda tienen sin embargo un alcance empírico bastante más amplio, pues pueden ser válidas también para sistemas jurídicos premodernos e incluso para sistemas normativos de carácter no jurídico» (p. 7).

2 «Una teoría del permiso y de la obligación, por ejemplo, al poder valer para todos los ordenamientos jurídicos posibles, tiene un alcance empírico más amplio que una teoría de los derechos sujetivos, que histórica y espacialmente es bastante más restringida, y ésta a su vez tiene un alcance más amplio que una teoría de los derechos fundamentales o únicamente de los derechos sociales» (Principa Iuris, I, p. 6).

3 Me refiero a la teoría del garantismo desarrollada por L. FERRAJOLI, 1995: Derecho y Razón. Teoría del galantismo penal, trad. cast. de P. ANDrÉs, J. C. BAyón, R. CANTARERO, A. Ruiz Miguel y J. TerRAdillos, Madrid: Trotta. 
tanto las posibles lagunas jurídicas que expresan los márgenes de inefectividad de las normas de nivel superior. La aproximación realista, por su parte, es también parcial o incompleta porque da cuenta del Derecho que (efectivamente) «es» pero ignora el derecho que debe ser. Ignora por tanto las posibles antinomias jurídicas que expresan los márgenes de ilegitimidad de las normas de nivel inferior. En otras palabras, en el Estado constitucional de Derecho siempre subsiste una virtual divergencia entre el «deber ser» constitucional del Derecho y su «ser» legislativo, y la teoría del Derecho debe estar en condiciones de dar cuenta de las antinomias y de las lagunas expresadas por esa virtual divergencia.

En el planteamiento de la metateoría semántica del Derecho de Principia Iuris, la divergencia entre el ser y el deber ser en el Derecho ha de ser, pues, explicitada y tematizada por la teoría del derecho. Es más, esta divergencia es el «objeto privilegiado de la teoría $»^{4}$. Por eso la teoría del Derecho integra estas aproximaciones sólo parciales al Derecho (la normativista y la realista) y para ello introduce, junto a la distinción entre los conceptos de vigencia o existencia y efectividad, también la distinción entre los conceptos de vigencia y validez.

La distinción entre vigencia y efectividad es la que media entre Derecho y realidad, entre normas y hechos; entre deber ser jurídico (o de derecho) y el ser no jurídico o de hecho. Se manifiesta en la inefectividad del Derecho y traduce, por consiguiente, la diferencia entre la perspectiva normativista y la realista. Por su parte, la distinción entre vigencia y validez es la que media entre el «ser» y el «deber ser» de las normas en el Derecho, y se vincula al constitucionalismo por cuanto sistema que incluye límites y vínculos (formales y sustanciales) a la producción legislativa. Se manifiesta en la invalidez del Derecho, o sea en la inefectividad de las normas que imponen tales límites. Por eso la validez (o invalidez) de las normas de nivel inferior equivale a la efectividad (o inefectividad) de las normas de nivel superior, y por tanto a la conformidad (o disconformidad) — en cuanto a la forma — o a la coherencia (o incoherencia) — en cuanto al significado- de las primeras respecto a las segundas.

Resulta claro, pues, que una teoría del Derecho construida conforme a estas categorías (vigencia o existencia, validez y efectividad) es al mismo tiempo normativista y realista, pues está en condiciones de explicar la doble naturaleza, normativa y fáctica, de los fenómenos jurídicos. Se supera así la tradicional identificación kelseniana y hartiana entre validez y existencia jurídica de las normas, que se muestra incapaz de dar cuenta del fenómeno de la existencia de normas inválidas. En el caso de KELSEN porque las normas se asumen como válidas (mientras no sean declaradas inválidas por un procedimiento de anulación) sólo porque son existentes. En el caso de HART porque, al identificar igualmente la validez de las normas con su existencia y hacerla depender del respeto por los límites a la competencia normativa, las normas se asumen como inexistentes sólo porque son inválidas. En ambos casos se descuida la doble vinculación, formal pero también sustancial, de los poderes normativos en el Estado constitucional de Derecho, y en consecuencia se impiden la comprensión y la crítica de ese fenómeno central en este modelo de Estado que es el Derecho inválido o ilegítimo5. La distinción

\footnotetext{
4 Principa Iuris, I, p. 14.

5 Principia Iuris, I, pp. 499-500.
} 
entre existencia o vigencia y validez permite tal comprensión y tal crítica, y propone así una imagen más completa y compleja del fenómeno jurídico.

\subsection{Distinción de la teoría frente a la dogmática, la sociología jurídica y la filosofía de la justicia: su carácter formal.}

Esta duplicidad de puntos de vista que asume la teoría del Derecho (el normativista y el realista) constituye el rasgo específico de su semántica frente a la semántica de cualquier otra teoría ${ }^{6}$, y en particular frente a la dogmática jurídica y a la sociología del Derecho, la primera de las cuales sólo adopta la perspectiva normativista que asume como referente empírico las normas mientras que la segunda sólo adopta la perspectiva realista que asume como referente empírico los hechos. En todo caso, además de estas diferencias semánticas existe otra diferencia esencial entre el discurso de la teoría del Derecho y los discursos de la dogmática y la sociología jurídicas; una diferencia que se da también frente al discurso de la filosofía política y que puede resumirse así: mientras que la teoría del Derecho tiene carácter puramente formal los otros tres discursos tienen carácter crítico.

Aunque todos estos discursos comparten un mismo objeto (el Derecho) y gran parte del aparato conceptual teórico, hay en efecto una diferencia entre la teoría del Derecho, por un lado, y la dogmática jurídica, la sociología del Derecho y la filosofía políti$\mathrm{ca}$, por otro. Estas tres últimas son discursos que desempeñan un papel crítico en relación con el Derecho: crítica de los eventuales perfiles de injusticia del Derecho en su conjunto, en el caso de la filosofía política; crítica de los eventuales perfiles de invalidez de las normas jurídicas, en el caso de la dogmática jurídica; y crítica de los perfiles de inefectividad del Derecho, en el caso de la sociología jurídica. Frente a estos discursos críticos, la teoría del Derecho se presenta como una «teoría formal que se limita al análisis de los conceptos teórico-jurídicos y de sus relaciones sintácticas», o si se quiere, al «análisis de las formas y de las estructuras del derecho positivo» ${ }^{7}$. Sus tesis y definiciones, por tanto, nos dicen qué son las normas, las prohibiciones, la validez, las obligaciones, la nulidad, etc., pero no nos dicen cuáles son en cada sistema concreto, ni cuáles deben ser, ni cómo son (o no son) de hecho realizadas. Son tesis y conceptos, en este sentido, ideológicamente neutrales, independientes de cualquier sistema de valores ${ }^{8}$.

Esta caracterización de la teoría del Derecho - probablemente discutible en lo que se refiere a su neutralidad' - tiene la indudable virtud de configurarla como un siste-

\footnotetext{
6 Principia Iuris, I, p. 11.

Principia Iuris, I, pp. 19 y 4.

Principia Iuris, I, p.19.
}

Algunos de sus conceptos, en efecto, parecen «construidos» o «reconstruidos» con el propósito de poder derivar a partir de ellos tesis valorativas concretas. Considérese, por ejemplo, la definición de «acto ilícito» como «acto informal prohibido» a la que nos referiremos en el siguiente epígrafe. Qué duda cabe que el «pragmatismo» de esa redefinición no depende sólo de su mayor capacidad explicativa y reconstructiva del fenómeno jurídico, sino también (y diría que sobre todo) de su mayor aptitud para realizar ciertos ideales. Tal opción es la única que permite afirmar el carácter prescriptivo (y en este sentido la relevancia) de muchas obligaciones y prohibiciones constitucionales e internacionales para las que no se han previsto sanciones para su incumplimiento e identificar la falta de las normas sancionatorias como un vicio (una laguna indebida) del ordenamiento. El propio FERRAJOLI confirma la dimensión valorativa de estas «elecciones» conceptuales: califi- 
ma de conceptos y de aserciones teóricas interpretable, bien por el discurso de la dogmática jurídica (si se toman como referencias empíricas de tales conceptos y aserciones las normas jurídicas que regulan los comportamientos humanos), bien por la sociología del Derecho (si se toman como referencias empíricas de la teoría del Derecho los comportamientos humanos regulados por las normas), o bien por el discurso filosófico político (si se valora y/o proyecta el derecho desde el punto de vista de un sistema axiológico dado). La «indudable virtud», pues de este modo FERRAJOLI ofrece un modelo integrado de ciencia jurídica en el que la teoría del Derecho representa la columna vertebral. En palabras del autor: la teoría del Derecho tiene carácter formal, pero, en el plano intensional, «es susceptible de una interpretación semántica de tipo realista, es decir desde el punto de vista descriptivo externo de la efectividad por parte de la sociología del Derecho; de una interpretación semántica de tipo normativista, es decir desde el punto de vista jurídico interno de la validez por parte de la dogmática jurídica; y de una interpretación semántica de tipo axiológico, es decir desde el punto de vista prescriptivo externo de la justicia por parte de la filosofía política ${ }^{10}$. La teoría del Derecho, en suma, es el lugar de encuentro de las diversas aproximaciones al estudio del Derecho, a las que ofrece un aparato conceptual en gran medida común.

\section{LA PRAGMÁTICA DE LA TEORÍA: SU PAPEL NORMATIVO Y NO MERAMENTE DESCRIPTIVO}

Cabría plantear en todo caso si una teoría del Derecho así caracterizada tiene sentido, o mejor, si es necesaria y en esta medida está justificada; es decir, en tanto que discurso lingüístico sobre el Derecho, la teoría ha de ser también evaluada desde su dimensión pragmática: para qué sirve o cuál es su objetivo. Es importante tener en cuenta a este respecto que, pese a su carácter elástico (o sea, su aptitud para reconstruir sistemas jurídicos en distintos grados de evolución y complejidad), la finalidad u objetivo central de la teoría (su «objeto privilegiado») es redefinir el paradigma teórico y normativo de las actuales democracias constitucionales, hoy en crisis, e identificar las garantías que permitan asegurar, frente a los diversos poderes, su máximo grado de efectividad para la tutela de los derechos. Y si éste es su objetivo prioritario cabe decir que está justificada; o más exactamente, que tiene sentido (o está justificado) perseguir este objetivo.

La teoría formal del Derecho, en efecto, elaborada como una disciplina autónoma y sistemática sobre todo a raíz de la obra de H. KELSEN, se asentaba sobre una serie de presupuestos metateóricos que hasta hace poco han coincidido con las características estructurales del Derecho positivo moderno (el principio de legalidad como norma de reconocimiento y fuente de legitimación del Derecho vigente; el monopolio estatal y le-

\footnotetext{
car como ilícitos la guerra y las violaciones de derechos fundamentales cometidas por los Estados «es el presupuesto jurídico de la construcción de una democracia internacional; del mismo modo que configurar las sanciones para tales ilícitos (...) como técnicas de garantía de los principios violados por ellos permite concebir su ausencia como "lagunas" que la comunidad internacional tiene la obligación de colmar». Y aún más explícitamente: a veces, en tales elecciones, «están presentes opciones de carácter ético-político» (Principia Iuris, I, pp. $475-476$ y p. 23).

10 Principia Iuris, I, p. 20. Véanse también pp. 39-43.
} 
gal de la producción jurídica; la separación de los poderes públicos, etc.). En los últimos tiempos, sin embargo, tales presupuestos han cambiado. Por muchos factores: por la ruptura del viejo nexo entre Derecho y Estado, que ha destruido la unidad e incrementado la incoherencia de los sistemas; por la ampliación de los asuntos en los que el Derecho interviene; por el surgimiento de nuevas desigualdades ligadas a la nacionalidad y ciudadanía; por la crisis de la razón jurídica y política y de la capacidad regulativa del Derecho, etc. Ahora bien, que los presupuestos de la teoría del Derecho hayan cambiado no significa que ésta haya dejado de tener sentido. Más bien al contrario: ha aumentado su necesidad como espacio autónomo para repensar y redefinir las categorías jurídicas teniendo en cuenta las nuevas formas del poder y las nuevas funciones del Derecho $^{11}$. La teoría del Derecho de Principia Iuris, precisamente, pretende reconstruir el modelo teórico y normativo del Estado constitucional de Derecho a fin de que resulten muy claros sus principios y bien visibles las divergencias entre las prácticas jurídicas y dichos principios. En cumplir este papel cifra la teoría su principal justificación.

La oportunidad de la teoría del Derecho se muestra, por otra parte, en su carácter pragmático y no meramente descriptivo. En efecto, la teoría del Derecho es pura o formal, en el sentido de que no dice nada sobre lo que los concretos sistemas jurídicos dicen o es justo que digan, pero esto no significa que no tenga un valor pragmático que se vincula al carácter artificial o convencional del Derecho. Este es uno de los presupuestos metateóricos centrales de Principia Iuris: el Derecho no es un objeto natural del que quepa dar cuenta con un discurso meramente descriptivo y avalorativo. Es un universo simbólico de creación humana, y en su construcción o «recreación» juegan un papel importante la teoría y la reflexión filosófica. El horizonte del jurista - dice FERRAJOLI- «no es el del simple espectador. Formamos parte del universo que describimos y contribuimos a producirlo con nuestras propias teorías» ${ }^{12}$. Esta dimensión pragmática de la teoría constituye una de las claves de su razón de ser.

La dimensión pragmática de la teoría se refleja en varios aspectos:

a) En primer término, los conceptos y tesis primitivas de la teoría son estipulados y asumidos por el teórico, en definitiva «elegidos» o «decididos», en función de criterios pragmáticos: sobre todo en función de su capacidad para explicar y reconstruir el derecho, particularmente — no importa insistir en ello- el derecho de las democracias constitucionales.

El propio FERRAJOLI pone un ejemplo que resulta bien expresivo de lo que se acaba de decir. La definición de «acto ilícito» como «acto informal prohibido», como alternativa a la definición kelseniana que entiende por ilícito el acto al que se conecta una sanción, no es gratuita sino que obedece a las diferentes implicaciones sobre el plano teórico y a los diferentes efectos sobre el plano práctico que tienen ambos conceptos. La definición propuesta por FERRAJOLI permite, por ejemplo, configurar como ilícitos los incumplimientos por parte del Estado de las obligaciones correspondientes a los derechos sociales, las violaciones por parte de los Estados de los derechos humanos establecidos por las cartas internacionales y desde luego la guerra, que no puede ser confi-

11 Precisamente con un repaso de todos estos fenómenos de crisis que acentúan hoy la necesidad de una nueva teoría del Derecho se abre la Introducción de Principia Iuris (véanse pp. 3 y 4).

12 Principia Iuris, I, p. 38. 
gurada como ilícito según la definición kelseniana si no es sosteniendo la extraña tesis de que «el bellum iustum, esto es la guerra en respuesta a una violación previa del Derecho internacional, constituye en sí mismo una sanción» ${ }^{13}$. En este sentido, pues, la reformulación de los términos primitivos de la teoría no es totalmente neutral, sino que está guiada por criterios pragmáticos. Y más exactamente por criterios que, en el fondo, están al servicio de la afirmación del paradigma constitucional y de la filosofía política que le sirve de base.

b) El valor pragmático de la teoría (y por tanto normativo o no puramente descriptivo) se muestra, en segundo término, en el papel normativo de los principios teóricos desarrollados por ella. La teoría explicita y reconstruye los principios que reflejan la estructura normativa del Derecho (unidad, coherencia y plenitud) y en esta medida impone al Derecho la satisfacción de tales principios lógicos. Más exactamente, estos principios permiten (o imponen) a la dogmática, por un lado, denunciar las antinomias y las lagunas y, por otro, instar su superación: la teoría desempeña de este modo un papel garantista en relación con el Derecho. Puede decirse en este sentido que las relaciones lógicas elaboradas por la teoría la hacen normativa en relación con el Derecho ${ }^{14}$, aunque ese carácter normativo no comporta un compromiso ideológico o valorativo.

c) Por lo demás, los paradigmas jurídicos son en gran parte el producto de las teorías y, en general, de las doctrinas jurídicas y políticas. Es decir, precisamente porque el Derecho es esencialmente un universo simbólico, la teoría produce imágenes y concepciones globales del derecho como «es», pero también, y sobre todo, del Derecho como «debe ser». En este sentido la teoría desempeña un papel por así decir constituyente o constructivo.

La teoría del Derecho de Principia Iuris, en suma, no es una teoría descriptiva que pretenda dar cuenta del Derecho realmente existente. Si ésta fuera su pretensión sería - al menos en algunos aspectos- manifiestamente falsa. Es una teoría formal que pretende reconstruir, redefinir o recrear ese constructo artificial que es el derecho positivo, y fundamentalmente el derecho positivo de las actuales democracias constitucionales. Bajo este aspecto la teoría tiene una incuestionable dimensión pragmática que se proyecta en la elección apropiada (encaminada al cumplimiento de ese objetivo) de sus conceptos y tesis primitivas y en el papel normativo o proyectivo que desempeñan sus principios teóricos en relación con el Derecho. Evidentemente esta dimensión pragmática de la teoría suele ser contestada por cuantos se empeñan en seguir fieles al viejo método descriptivista y avalorativo del Derecho. A juicio de FERRAJOLI, sin embargo, este empeño obedece a la ilusión paracientífica — y equivocada - de que el Derecho puede ser estudiado como un objeto natural, autónomo frente a la política y a la reflexión teórica y filosófica. Superada esta ilusión aparece inevitablemente la aludida dimensión pragmática de la teoría y de la ciencia del Derecho: el Derecho no es ajeno a nuestras reflexiones, «es como lo pensamos, lo teorizamos, lo proyectamos, lo producimos, lo interpretamos, lo defendemos y lo transformamos, de modo que todos tenemos una parte de responsabilidad en cómo es» ${ }^{15}$.

\footnotetext{
13 Principia Iuris, I, pp. 23 y 475.

14 Principia Iuris, I, p. 33.

15 Principia Iuris, I, p. 38.
} 


\section{LA SINTÁCTICA DE LA TEORÍA: SU AXIOMATIZACIÓN}

«Este libro contiene una teoría del Derecho construida con el método axiomático». Con esta clara y contundente afirmación se abre el Prefacio de Principia Iuris.

Axiomatizar la teoría supone reelaborar todo el lenguaje jurídico y fundamentar sus aserciones. Más precisamente, supone, primero, asumir conceptos primitivos y tesis primitivas (postulados o definiciones) que — como se ha dicho- deben ser justificados en función de criterios pragmáticos; y, después, definir todos los demás conceptos a partir de otros conceptos teóricos y mediante reglas de formación previamente establecidas, y demostrar todas las demás tesis a partir de otras tesis teóricas y mediante reglas de transformación también preestablecidas ${ }^{16}$. La teoría, por tanto, estará justificada desde el punto de vista sintáctico si puede demostrarse, mediante operaciones lógicas controlables, que todas las tesis derivadas (teoremas) se deducen de las tesis primitivas (postulados o definiciones) o de otras tesis a su vez derivadas. El método axiomático, el cálculo, hace el discurso prolijo y farragoso, pero tiene la virtud de que permite o facilita ese control.

Por lo demás, que el libro se abra con una afirmación como la trascrita más arriba sobre la sintaxis de la teoría no es baladí. Pone de relieve que la axiomatización de la teoría es uno de sus rasgos centrales, sino incluso el más importante. Esto es así porque la formalización de la teoría no sólo permite una mayor transparencia y control lógico de las tesis que contiene, sino que además, y como consecuencia de ello, contribuye también a hacer más visible la divergencia entre ser y deber ser, entre efectividad y normatividad, que como se ha dicho constituye el objeto privilegiado de la teoría. $\mathrm{Na}$ turalmente toda teoría del Derecho, y también por tanto la teoría del Derecho de Principia Iuris, puede ser construida sin recurrir a su formalización. De hecho — como el mismo autor reconoce- el empleo del método axiomático es el aspecto más llamativo e insólito de la teoría que desarrolla. Por eso, y dado que el propio FERRAJOLI confiesa que - desde el punto de vista metateórico- la teoría formalizada refleja una aspiración de la filosofía de la ciencia del neopositivismo lógico presente aún en los años sesenta del pasado siglo, que es cuando Principia Iuris fue proyectado, cabría preguntarse si el autor, en el caso de tener que proyectar hoy esa teoría, la hubiese planteado de la misma manera, o sea, si la hubiese concebido también como una teoría axiomatizada o formalizada.

\section{EL RENDIMIENTO O LA ADECUACIÓN DEL SISTEMA TEÓRICO DE PRINCIPIA IURIS}

La mayor o menor adecuación del sistema teórico de Principia Iuris dependerá, pues, de tres cosas:

1. De que sea una buena reconstrucción de los sistemas jurídicos más complejos, particularmente de los que se ajustan a la fórmula del Estado constitucional de Derecho; es decir, de que resulte adecuado desde el punto de vista semántico.

16 Principia Iuris, Prefacio, p. VI. 
2. De que sus tesis primitivas (postulados y definiciones) tengan no sólo capacidad de explicación analítica o sistemática sino también fecundidad crítica o proyectiva; es decir, de que sea adecuado desde el punto de vista pragmático.

3. De que todas las tesis derivadas a partir de las tesis primitivas sean coherentes con éstas, lo que equivale a decir que pueda haber un control lógico de todas las operaciones mediante las cuales unas tesis puedan ser demostradas a partir de otras; es decir, de que resulte adecuado desde el punto de vista sintáctico.

Obsérvese a este respecto que, tanto desde el punto de vista semántico como sobre todo desde el punto de vista pragmático, una pretensión básica de esta teoría es dar cuenta de la insuprimible divergencia entre principios y praxis jurídica, entre ser y deber ser, efectividad y normatividad, y por consiguiente dar cuenta de los perfiles de inefectividad de los principios y de ilegitimidad de la praxis. La teoría de la divergencia representa, pues, el nervio central del paradigma teórico y normativo del Estado constitucional que constituye el objeto privilegiado de la teoría. Ese paradigma estaba ya anunciado en sus líneas básicas en Derecho y razón ${ }^{17}$, y por tanto la adecuación de la teoría, tanto desde el punto de vista semántico como pragmático, de alguna manera ha sido ya objeto de análisis en los múltiples debates que se produjeron a raíz de la publicación de aquella obra ${ }^{18}$; y lo será — sin duda con mayor razón- a partir de la publicación de ésta. El gran salto de Principia Iuris, la novedad radical, es la utilización del método axiomático en su reconstrucción. Es más, la teoría —como dice su autor- es justamente (y sólo) la expresada por las fórmulas, por el cálculo. El resto es traducción y comentario ${ }^{19}$.

Este trabajo de axiomatización ha supuesto un esfuerzo admirable y titánico que pone de relieve el afán de FERRAJOLI por construir una ciencia jurídica more geometrico demonstrata. Obviamente podrá discutirse el mayor o menor rendimiento de este esfuerzo; por ejemplo, cabría cuestionar la función práctica que el autor atribuye a la axiomatización, y en particular se podría poner seriamente en duda —en contra de lo que se afirma ${ }^{20}$ - que la axiomatización mediante la reconstrucción de todo el lenguaje teórico haga que la teoría también resulte accesible a los no expertos en derecho. De lo que no cabe duda, sin embargo, es de que tras este esfuerzo se vislumbra el compromiso con la herencia ilustrada, compromiso que se muestra en la aspiración —como afirma el autor en el Prefacio- a «la simplificación del lenguaje teórico, a la claridad y univocidad de los conceptos y las tesis sostenidas, a la exposición al control lógico de todas las deducciones y a la crítica extra-lógica de todas las asunciones; a la coherencia y sistematicidad del conjunto; $y$, en fin, a la superación de las ambigüedades y de las muchas aporías y paralogismos que son inevitables en cualquier discurso formulado en lenguaje común ${ }^{21}$. Estas son las virtudes teoréticas de la formalización de la teoría, y desde luego no parece que puedan ser seriamente cuestionadas.

17 Me refiero, claro está, a Derecho y Razón. Teoría del garantismo penal, cit.

18 Entre los múltiples análisis de los que la obra mencionada ha sido objeto me permito remitir a dos obras colectivas: 1993: Le ragioni del garantismo. Discutendo con Luigi Ferrajoli, L. GIAFORMAGGIO (ed.), Torino: Giappichelli; y 2005: Garantismo. Estudios sobre el pensamiento jurídico de Luiggi Ferrajoli (M. CARBONELL y P. SALAZAR, coords.), Madrid: Trotta-UNAM.

19 Principia Iuris, I, Prefacio, p. VIII.

${ }_{20}$ Principia Iuris, I, Prefacio, p. VII.

${ }^{21}$ Principia Iuris, I, Prefacio, pp. VI-VII. 
Lo que a mi juicio puede parecer paradójico, o por lo menos llama la atención, no es tanto esa apuesta metateórica hiperracionalista cuanto su combinación, por una parte, con una concepción del poder que desconfía profundamente de su racionalidad y, por otra, con una praxis política y jurídica que es, de hecho, irracional e ilegal; en algunos aspectos profundamente irracional e ilegal. Esa paradoja, sin embargo, es sólo aparente. La concepción escéptica y realista del poder, y la cada vez mayor ausencia de racionalidad de los sistemas jurídicos que conocemos y que se inspiran en el paradigma constitucionalista, no hace inadecuada una construcción hiperracional de este paradigma. Al contrario. Precisamente porque se desconfía del poder y se confía al Derecho la articulación de un sistema de vínculos efectivos a su ejercicio para la protección de los bienes y derechos que deban ser perseguidos, el Derecho - o mejor, el principio de legalidad- es visto como un instrumento civilizador y el Estado constitucional de Derecho como la máxima expresión de ese principio. Por eso lo que la teoría debe hacer es reconstruir ese paradigma del modo más claro y preciso posible, a fin de que, desde el mismo, puedan ponerse de relieve y criticarse las desviaciones de la praxis. La desconfianza hacia el poder, en suma, justifica la apuesta por el modelo del Estado constitucional de Derecho, como ya quedaba claro en Derecho y Razón. Y la teoría del Derecho expresada en Principia Iuris reconstruye ese modelo de modo hiperracionalista no sólo por las virtudes teoréticas que ello supone sino también para poder medir mejor la distancia que separa la realidad del modelo e instar — en su caso- a la transformación de la misma. FERRAJOLI inserta así la «lucha por la justicia» en el propio seno de la teoría. Y esto puede hacerlo porque la teoría no es un discurso meramente descriptivo sino reconstructivo del universo lingüístico del Derecho.

Cabría decir pues, en conclusión, que Principia Iuris representa en cierto modo la continuación lógica de Derecho y Razón y es por ello, en última instancia, fiduciario de sus mismos postulados ilustrados. Frente a algunas teorizaciones de gran predicamento en los últimos tiempos que ven en el Derecho una obra colectiva y cooperativa y que parecen asumir implícitamente una gran confianza en la bondad del poder o de la anomia, la teoría general del garantismo arrancaba de la idea — presente ya en LOCKE y en MONTESQUIEU - de que del poder hay que esperar siempre un potencial abuso ${ }^{22}$ que es preciso neutralizar haciendo del Derecho un sistema de garantías, de límites y vínculos al poder para la tutela de los derechos ${ }^{23}$. Por eso el garantismo se opone al autoritarismo en política y al decisionismo en Derecho, propugnando, frente al primero, la democracia sustancial y, frente al segundo, el principio de legalidad; y por eso el estado constitucional de Derecho es visto como el producto de la mejor ingeniería jurídica. La teoría del Derecho de Principia Iuris es la argamasa que cimenta esa visión. Su reconstrucción racional del modelo del Estado constitucional de Derecho pretende servir ahora como instrumento para el análisis de los consiguientes niveles de inefectividad y de ilegitimidad (interna y externa) de los sistemas jurídicos que a él se remiten.

22 «La libertad política... aparece... sólo cuando no se abusa del poder. Pero es una experiencia eterna que todo hombre que tiene poder siente la inclinación de abusar de él», 1972: Del espíritu de las leyes (1748), trad. de M. BlázQueZ y P. DE VegA, Madrid: Tecnos, Libro XI, cap. IV, p. 150.

23 Precisamente el carácter potencialmente abusivo del poder es lo que llevó a MONTESQUIEU a configurar el concepto de límite como factor esencial para asegurar la tutela de la libertad, y de ahí la necesidad de la «separación de poderes» que habrá de conseguirse a través de un sistema de frenos y contrapesos entre ellos. Véase R. BLANCO, 1994: El valor de la Constitución, Madrid: Alianza, pp. 67 y ss. 
Y su construcción axiomatizada, en la medida en que pueda hacer más visibles los déficit de racionalidad de la praxis, pretende contribuir también a ese objetivo. Cabe dudar, desde luego, de que la axiomatización sea el mejor camino para cumplir ese objetivo de transparencia, de visibilidad de las divergencias y de controlabilidad de los déficit de racionalidad de la praxis, pero de lo que no cabe dudar es de que dicho objetivo - al menos para quien participe de una visión negativa del poder y positiva del Derecho- está sobradamente justificado. Principia Iuris, en suma, tal vez adolece de una más completa filosofía moral que legitime las opciones valorativas que están en la base de la teoría, pero tiene detrás una concepción del poder que resulta a mi juicio sumamente atractiva y en todo caso muy higiénica. Si hace unos años M. JorI decía, en referencia a la teoría general del garantismo de Derecho y Razón, que de ella podrá disentirse en todo o en parte pero que ya no se podrá prescindir ${ }^{24}$, creo que lo mismo cabe decir ahora de la teoría del Derecho de Principia Iuris: se la podrá criticar, pero difícilmente se la podrá obviar.

${ }^{24}$ M. JORI, «La cicala e la formica», en Le ragioni del garantismo. Discutendo con Luigi Ferrajoli, cit., p. 66. 